\title{
Synthesis and conformation of short peptides modeled after peptide LL-37
}

\author{
Barbara Biondi $^{1}$, Silvia Millan ${ }^{2}$, Fernando Formaggio $^{2}$, Alessandra Semenzato $^{3}$, Cristina Peggion ${ }^{2}$ \\ ${ }^{1}$ ICB, Padova Unit, CNR, Department of Chemistry, University of Padova, Italy, Italy \\ ${ }^{2}$ Department of Chemistry, University of Padova, Italy \\ ${ }^{3}$ Department of Pharmaceutical Sciences, University of Padova, Italy
}

https://doi.org/10.17952/35EPS.2018.195

\section{Introduction}

Numerous tissues and cells of our body (e.g., epithelial or innate immune cells) are able to produce the 37residue peptide cathelicidin LL-37 [1]. LL-37 plays important roles in the innate immune system as it acts as a natural antibiotic against Gram-positive and Gram-negative bacteria, fungi, and even viruses. In addition, it may inhibit the viral replication, promote wound healing [1,4] and kill tumor cells $[1,2,3,4,5]$. LL-37 is an amphipathic, $\alpha$-helical peptide with an overall positive charge $(+6)$. The helical structure promotes cell killing by binding to the negatively-charged bacterial membrane. Its possible mechanisms of action, based on the disruption of cell membranes, were recently reviewed $[3,6]$.

Alteration of LL-37 were observed to occur in some pathologies, as for instance psoriasis (overproduction of LL-37) and topic dermatitis (inability to produce LL-37).

\section{Results and Discussion}

To make LL-37 industrially appealing as a drug, we started a program aimed at identifying shorter segments with the same potency of LL-37. We synthesized short, helical peptides stabilized by one or two helix-promoting Aib ( $\alpha$-aminoisobutyric acid) residues:

1 Pal-RKSKEKIG-NH $2(\mathrm{Pal}=$ palmitoyl $)$

\section{Pal-KSKEKIG-NH 2}

3 Ac-RKUKEKIG-NH $2($ Ac = acetyl; $\mathrm{U}=\mathrm{Aib})$

4 Pal-RKUKEKIG-NH 2

\section{Ac-KRUVQRUKDFLR-NH 2}

\section{Pal-KRUVQRUKDFLR-NH ${ }_{2}$}

Peptides 1 and 2 correspond to segments 7-14 and 8-14, respectively, of LL-37. To protect them from the enzymatic degradation we added at the N-terminus a palmitoyl moiety, able also to promote membrane insertion. In peptides 3 and 4 the helix-promoting and hydrophobic Aib residue replaces a Ser9. We chose this substitution after having observed that the hydrophilic $\operatorname{Ser}^{9}$ is located on the hydrophobic face of the $\alpha$-helical LL-37. With peptides 5 and 6 we focused on the central part of LL-37, segment 18-29, known to be quite active. Also in this case we aimed at assessing the possible beneficial effects of palmitoylation and Aib insertion.

All peptides were synthesized by the solid phase approach and purified by reverse-phase HPLC. Their 3Dstructures were assessed by means of circular dichroism, 2D-NMR and FT-IR absorption. For all peptides we observed $\alpha-/ 3_{10}$-helix equilibria, strongly dependent on the solvent used (see Figure 1 as an example). The substitution of Ser with Aib led to rather stable helices in membrane-mimetic environments (SDS and TFE). Interestingly, peptide 1 adopts a helical conformation although lacking any helix-promoting Aib residue. The presence of the $\mathrm{N}$-terminal palmitic chain does not appear to affect the helical conformation.

Preliminary experiments of interactions with model membranes reveal for peptide 4 an interesting selectivity towards eukaryotic membrane mimics (Figure 2). Peptide 1, having the same sequence as 4 but with Ser instead of Aib, has no interaction with both type of vesicles (Figure 2). Therefore, the insertion of Aib appears to be a promising tool to modulate membrane selectivity and, hopefully, bioactivity. 


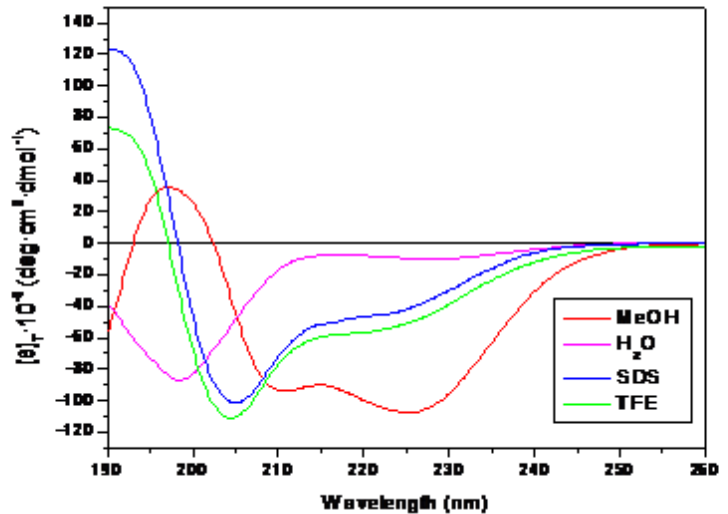

Figure 1: Circular dichroism of peptide 3, Ac-RKUKEKIG-NH $\mathrm{H}_{2}$, in different solvents.
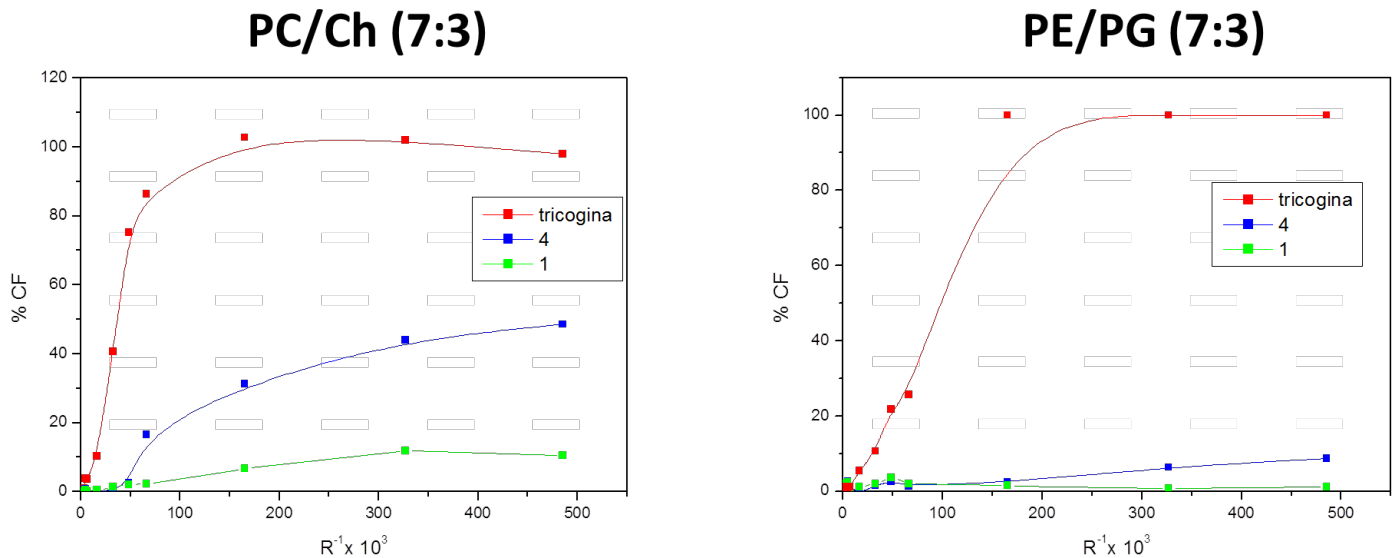

Figure 2: Peptide-induced carboxyfluorescein leakage from artificial small unilamellar vesicles (SUV), as a function peptide concentration. Left: SUV mimicking eukaryotic cells, (7:3) egg phosphatidylcholine/cholesterol; right: SUV mimicking bacterial cells, (7:3) phosphatidylethanolamine/phosphatidylglycerol. The fractional releases were determined as a function of the ratio $R=[$ peptide]/[lipid] (x10-3) 20 minutes after peptide addition.

\section{References}

[1] P. Chieosilapatham, S. Ikeda, H. Ogawa, F. Niyonsaba, Cur. Pharm. Design 2018, 24, 1079-1091. DOI: $10.2174 / 1381612824666180327113418$

[2] M. E. Pachón-Ibánez, Y. Smani, Jerónimo Pachón, J. Sánchez-Céspedes, FEMS Microbiol. Rev. 2017, 41, 323-342. DOI: 10.1093/femsre/fux012

[3] K. Zeth, E. Sancho-Vaello, Front. Chem. 2017, 5, art. 86. DOI: 10.3389/fchem.2017.00086

[4] A. A. Baxter, F. T. Lay, I. K. H. Poon, M. Kvansakul, M. D. Hulett, Cell. Mol. Life Sci. 2017, 74, 3809-3825. DOI: $10.1007 / \mathrm{s} 00018-017-2604-\mathrm{Z}$

[5] E. Ogawa, Y. Sato, A. Minagawa, R. Okuyama, J. Dermat. 2018, 45, 264-272. DOI: $10.1111 / 1346-8138.14139$

[6] Z. Khurshid, M. Naseem, F. Y. I. Asiri, M. Mali, R. S. Khan, H. A. Sahibzada, M. S. Zafar, S. F. Moin, E. Khan, Biomolecules 2017, 7, art. 80. DOI: 10.3390/biom7040080 